\title{
Anestesia intravenosa total utilizando propofol ou propofol/cetamina em cadelas submetidas à ovariossalpingohisterectomia
}

\author{
Total intravenous anesthesia using propofol or propofol/ketamine in bitches undergoing \\ ovariohysterectomy
}

\author{
Simone Salata Gasparini' Stelio Pacca Loureiro Luna ${ }^{\text {II }}$ Renata Navarro Cassu ${ }^{\text {III }}$ \\ Fernando de Biasi ${ }^{\mathrm{IV}}$
}

\section{RESUMO}

Objetivou-se avaliar os efeitos cardiorrespiratório e analgésico da infusão contínua com propofol e propofol/ cetamina em cadelas pré-medicadas com atropina e xilazina, submetidas a ovariossalpingohisterectomia (OSH). Em seis cadelas (GP) a indução anestésica foi realizada com propofol (5mg kg-1 iv), seguido da manutenção anestésica com o mesmo fármaco em infusão contínua intravenosa na taxa inicial de $0,4 \mathrm{mg} \mathrm{kg}^{-1} \cdot \mathrm{min}^{-1}$. Outras seis cadelas (GPC) receberam a associação de propofol $\left(3,5 \mathrm{mg} \mathrm{kg}^{-1} \mathrm{iv}\right)$ e cetamina $\left(1 \mathrm{mg} \mathrm{kg} \mathrm{kg}^{-1}\right.$ iv) como indução anestésica. Depois, foi feita manutenção anestésica em infusão contínua intravenosa inicial com $0,28 \mathrm{mg}$ $\mathrm{kg}^{-1} \cdot \mathrm{min}^{-1}$ e $0,06 \mathrm{mg} \mathrm{kg}^{-1} \cdot \mathrm{min}^{-1}$ de propofol e cetamina, respectivamente. Os seguintes parâmetros foram mensurados durante a anestesia a cada 10 minutos: freqüências cardíaca (FC) e respiratória (f), pressão arterial sistólica, média e diastólica (PA), concentração final expirada de $\mathrm{CO}_{2}\left(\mathrm{EtCO}_{2}\right)$, volume minuto (VM), pressão parcial de gás carbônico $\left(\mathrm{PaCO}_{2}\right)$, pressão parcial de oxigênio $\left(\mathrm{PaO}_{2}\right)$, saturação de oxigênio na hemoglobina $\left(\mathrm{SatO}_{2}\right), \mathrm{pH}$, bicarbonato, glicemia e temperatura retal (T). Observou-se redução da pressão arterial média entre 20 e 40 minutos de anestesia no GP. Ocorreu redução da temperatura, hipercapnia e acidose respiratória em ambos os grupos durante a anestesia. A $\mathrm{PaO}_{2}$, o bicarbonato e a glicose aumentaram de forma significativa apenas no GPC durante a anestesia. Houve necessidade de aumentar em 50 e $20 \%$ a taxa de infusão de propofol no GP e GPC respectivamente para anestesia cirúrgica satisfatória. Dessa forma, ambos os protocolos mostraram-se seguros e suficientes do ponto de vista de anestesia cirúrgica para realização da OSH em cadelas, desde que a ventilação assistida ou controlada seja instituída quando necessária e a velocidade de infusão do propofol seja 0,6 e 0,34mg $\mathrm{kg}^{-1} \cdot \mathrm{min}^{-1}$ nos grupos GP e GPC, respectivamente.
Palavras-chave: infusão contínua, anestésico geral, anestésico dissociativo.

\begin{abstract}
This study aimed to investigate the cardiopulmonary and analgesic effects of propofol and propofol/ketamine infusion in bitches premedicated with atropine and xylazine and submitted to ovariohisterectomy. In six bitches, anesthesia was induced by $5 \mathrm{mg} \mathrm{kg}^{-1}$ of propofol and maintained initially with $0.4 \mathrm{mg} \mathrm{kg}^{-1}$. $\mathrm{min}^{-1}$ of propofol (GP). In the other six bitches, anesthesia was induced with a combination of $3.5 \mathrm{mg} \mathrm{kg}^{-1}$ propofol and $1 \mathrm{mg} \mathrm{kg}^{-1}$ of ketamine and maintained initially with $0.28 \mathrm{mg} \mathrm{kg}^{-1} \cdot \mathrm{min}^{-1}$ propofol and $0.06 \mathrm{mg} \mathrm{kg}^{-1} \cdot \mathrm{min}^{-1}$ of ketamine (GPK). Heart and respiratory rates, arterial blood pressure, minute ventilation, end tidal $\mathrm{CO}_{2}$, pulse hemoglobin $\mathrm{O}_{2}$ saturation, blood gas analysis, plasma glucose concentration and temperature were measured before and every 10 minutes during anesthesia. Mean arterial blood pressure reduced from 20 to 40 minutes of propofol anesthesia. Temperature reduction and hypercapnia with respiratory acidosis occurred in both groups during anesthesia. $\mathrm{PaO}_{2}$, bicarbonate and glucose increased only during propofol/ ketamine anesthesia. Propofol infusion rate was increased by 50 and 20\% during anesthesia in GP and GPK respectively to allow satisfactory surgical anesthesia. Both anesthetic protocols were safe and adequate for ovariohysterectomy in bitches, considering that controlled ventilation is performed when necessary and propofol infusion rate is adjusted to 0,.6 and $0.34 \mathrm{mg} \mathrm{kg}^{-1} \cdot \mathrm{min}^{-1}$ in GP and GPK according to the surgical stimulus.
\end{abstract}

Key words: infusion, general anaesthetic, dissociative anaesthetic.

IFaculdade de Medicina Veterinária e Zootecnia (FMVZ), Universidade Estadual Paulista (UNESP), Botucatu, SP, Brasil.

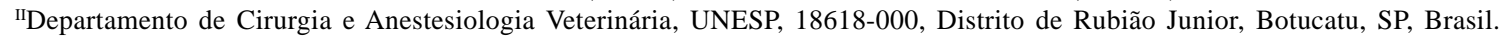

IIIDepartamento de Cirurgia e Anestesiologia Veterinária, Faculdade de Ciências Agrárias, Universidade do Oeste Paulista (Unoeste), Rodovia Raposo Tavares, km 572, Campus II, Bairro Limoeiro, 19067-175, Presidente Prudente, SP, Brasil. E-mail: renavarro@uol.com.br. Autor para correspondência.

IV Departamento de Clínicas Veterinárias, Faculdade de Medicina Veterinária, Universidade Estadual de Londrina (UEL), Londrina, PR, Brasil. 


\section{INTRODUÇÃO}

Muitos fármacos podem ser utilizados em anestesia intravenosa (MUIR, 1994; ILKIW, 1999, MASSONE, 2008). Entretanto, nenhum deles apresenta propriedades que permitam seu uso isoladamente para que se produza anestesia adequada (ILKIW, 1999; HEMERIJAK \& KISSIN, 2001). Portanto, a partir de conhecimentos farmacodinâmicos e farmacocinéticos, devem-se utilizar os fármacos mais indicados para cada situação, por meio de associação destes dentro da mesma técnica (MUIR, 1994), para se estabelecer a anestesia intravenosa total (TIVA).

Dentre esses fármacos, destaca-se o propofol, cuja utilização em infusão contínua é vantajosa em função da rapidez na indução (BULAFARI et al., 1995) e na recuperação (SMITH et al., 1994; GANEM et al., 2002), mesmo em caso de administrações prolongadas, já que a rápida redistribuição do fármaco para compartimentos periféricos traduz-se em pronto despertar (WATKINS et al., 1987; HALL \& CHAMBERS, 1987, MASSONE 2008), além de ser rapidamente metabolizado, principalmente pelo fígado, mas também em outros sítios metabólicos (COURT et al., 2001). No entanto, o propofol apresenta efeitos depressores sobre o sistema cardiorrespiratório, que podem ser minimizados pela redução da dose desse agente anestésico. Ademais, o propofol não tem propriedades analgésicas (BRANSON, 2007). Dessa forma a utilização de analgésicos como componentes da anestesia balanceada é justificada pela possibilidade da obtenção de melhor qualidade anestésica em relação ao uso isolado do propofol (O'CONNOR \& SEAR, 1988).

Os analgésicos podem ser administrados na medicação pré-anestésica, bem como durante a manutenção da anestesia, mediante infusão contínua (REDONDO et al., 1999). Efeito sinérgico tem sido relatado com o uso de alfa ${ }_{2}$ adrenoceptores agonistas na medicação pré-anestésica, favorecendo a redução da dose de indução e de manutenção anestésica do propofol(CULLEN\&REYNOLDSON, 1993; ENGLAND et al., 1996). Paralelamente, outros estudos têm demonstrado resultados satisfatórios com a infusão contínua de propofol associada a fármacos com potencial analgésico, como opióides (HATSCHBACH et al., 2008) e cetamina (MANNARINO, 2005) para obtenção de anestesia cirúrgica, com mínimos efeitos adversos (BRANSON, 2007). SELISKAR et al (2007) obtiveram valores mais elevados de freqüência cardíaca e pressão arterial média em cães anestesiados com cetamina/propofol quando comparados aos cães anestesiados somente com propofol, corroborando resultados prévios relatados por INTELIZANO et al (2008) os quais observaram maior estabilidade hemodinâmica em cães sob infusão contínua com propofol e cetamina em relação ao propofol isolado.

O objetivo desse estudo foi avaliar os efeitos cardiorrespiratórios, a qualidade da anestesia e a recuperação pós-anestésica da infusão de propofol e propofol/cetamina em cadelas pré-tratadas com atropina e xilazina submetidas à ovariossalpingohisterectomia.

\section{MATERIAL E MÉTODOS}

Foram utilizadas doze cadelas provenientes da rotina cirúrgica do Hospital Veterinário da Instituição de Origem, de diferentes raças, com peso médio de $10 \pm 0,6 \mathrm{~kg}$. Elas foram consideradas clinicamente saudáveis, após a realização de exames físico e laboratoriais, como hemograma, dosagem sérica de uréia, creatinina, enzimas alanino aminotransferase, aspartato aminotransferase e fosfatase alcalina. As cirurgias foram realizadas sempre pelo mesmo cirurgião, que desconhecia o protocolo anestésico empregado. Todos os animais foram submetidos a jejum alimentar de 12 horas e hídrico de seis horas, antes da realização do experimento. A medicação pré-anestésica (MPA) foi realizada mediante a administração subcutânea (sc) de atropina ${ }^{\mathrm{a}}$ na dose de $0,05 \mathrm{mg} \mathrm{kg}^{-1}$. Depois de 15 minutos foi realizada a administração intramuscular (im) de xilazina ${ }^{\mathrm{b}}$ na dose de $1 \mathrm{mg} \mathrm{kg}^{-1}$ e 10 minutos depois foi realizada a cateterização da veia cefálica direita, por onde foi injetado propofol ${ }^{\mathrm{c}}$ na dose de $5 \mathrm{mg} \mathrm{kg}^{-1}$ (GP) ou cetamina ${ }^{\mathrm{d}}$ na dose de $1 \mathrm{mg} \mathrm{kg}^{-1}$ em associação ao propofol na dose de 3,5 $\mathrm{mg} \mathrm{kg}^{-1}$ (GPC), separadamente nesta ordem, para indução anestésica. Durante a manutenção anestésica os fármacos foram infundidos isolados por cada uma das veias cefálicas, utilizandose bomba de infusão de seringa ${ }^{\mathrm{e}}$. A anestesia foi mantida com infusão contínua de propofol $\left(0,4 \mathrm{mg} \mathrm{kg}^{-1} \cdot \mathrm{min}^{-1}\right)$ ou cetamina $\left(0,06 \mathrm{mg} \mathrm{kg}^{-1} \cdot \mathrm{min}^{-1}\right)$ associada ao propofol $\left(0,28 \mathrm{mg} \mathrm{kg}^{-1} \cdot \mathrm{min}^{-1}\right)$, para os animais do GP e GPC, respectivamente. Após a indução da anestesia, foi realizada intubação endotraqueal em todos os animais, com administração de oxigênio a $100 \%\left(10 \mathrm{ml} \mathrm{kg}^{-1} \cdot \mathrm{min}^{-1}\right)$, com manutenção destes sob respiração espontânea. Realizou-se fluidoterapia com Ringer lactato $\left(10 \mathrm{ml} \mathrm{kg}^{-1} \cdot \mathrm{h}^{-1}\right)$ durante todo o procedimento anestésico, por meio de um cateter introduzido na veia jugular.

Para ambos os grupos, a anestesia foi conduzida com base nos efeitos anestésicos, visando à manutenção dos animais com ausência dos reflexos protetores e movimentos involuntários, além das variáveis cardiovasculares dentro dos valores fisiológicos para a espécie. Dessa forma, a taxa de 
infusão de propofol foi elevada ou reduzida, visandose à manutenção do plano anestésico suficiente para a realização do procedimento cirúrgico.

Foram avaliados freqüência cardíaca (FC) e ritmo cardíaco por meio de eletrocardiograma ${ }^{\mathrm{f}}$, com uso de eletrodos acoplados nos membros anteriores e posteriores com leitura na segunda derivação de Eithoven. Antes da indução anestésica a FC foi avaliada por meio de estetoscópiog. Já a saturação de pulso de oxigênio $\left(\mathrm{SatO}_{2}\right)$ e a concentração final expirada de $\mathrm{CO}_{2}$ $\left(\mathrm{ETCO}_{2}\right)$ foram avaliadas por meio de oxicapnógrafo ${ }^{\mathrm{h}}$, cujo sensor para oximetria foi adaptado na língua dos animais, e o sensor para capnografia foi adaptado lateralmente entre a sonda endotraqueal e a conexão do circuito anestésico. A freqüência respiratória $(f)$, o volume corrente (Vt) e o volume minuto (VM) foram avaliados por meio de ventilômetro, já a pressão arterial não invasiva sistólica (PAS), média (PAM) e diastólica (PAD) foi avaliada com monitor oscilométrico ${ }^{\mathrm{j}}$, pela adaptação do manguito pediátrico na região proximal do rádio. Utilizou-se manguito cuja largura equivalesse a $40 \%$ da circunferência do membro. A temperatura retal (T) foi mensurada pela introdução retal de termômetro digitalk $^{\mathrm{k}}$ e a hemogasometria ${ }^{1}$ pressão parcial de gás carbônico $\left(\mathrm{PaCO}_{2}\right)$, pressão parcial de oxigênio $\left(\mathrm{PaO}_{2}\right)$, saturação de oxigênio na hemoglobina $\left(\mathrm{SatO}_{2}\right), \mathrm{pH}$, bicarbonato e glicemia, pela cateterização ${ }^{\mathrm{m}}$ da artéria femoral. As amostras de sangue arterial $(0,5 \mathrm{ml})$ foram colhidas em seringas de insulina heparinizadas, sendo mantidas sob refrigeração entre 4 e $6^{\circ} \mathrm{C}$ por período máximo de três horas, sendo posteriormente encaminhadas ao Laboratório de Análises do Hospital das Clínicas da Instituição de Origem. O tempo cirúrgico foi avaliado em minutos e a qualidade da anestesia (VIEIRA, 1999) foi avaliada pelo cirurgião, por meio de escala analógica visual (0-10). Foram avaliados grau de analgesia e relaxamento muscular, utilizando uma linha de $10 \mathrm{~cm}$, de modo que o extremo esquerdo representava o animal sem sinais de desconforto e com excelente relaxamento muscular, enquanto o extremo direito representava grande sinal de desconforto e pobre relaxamento muscular que impossibilitasse a cirurgia. A recuperação pós- anestésica foi avaliada pelo anestesista, com registro do tempo necessário para ocorrência do primeiro movimento da cabeça, posicionamento em decúbito esternal e em posição quadrupedal. Durante o procedimento cirúrgico e o período pós-operatório os animais foram aquecidos pelo colchão térmico, até a obtenção do retorno da temperatura retal aos valores basais.

Antes do início do procedimento anestésico, 10 minutos após a administração da MPA, imediatamente após a indução da anestesia e a cada 10 minutos até o término do procedimento cirúrgico foram coletados os dados de FC, $f$, VM, Vt, PAS, PAM, PAD, $\mathrm{SatO}_{2} \mathrm{ETCO}_{2}$ e T. As variáveis $\mathrm{PaCO}_{2}, \mathrm{PaO}_{2}, \mathrm{SatO}_{2}, \mathrm{pH}$, bicarbonato e glicemia foram avaliadas nos mesmos momentos supracitados, com exceção do momento imediatamente após a indução anestésica.

A estatística foi realizada por meio de análise de variância, seguida pelo teste de Dunnett para avaliação de diferenças ao longo do tempo dentro de cada grupo e teste de Fisher para comparação das diferenças entre os grupos em cada momento. Considerou-se diferença significativa quando $\mathrm{p}<0,05$ (ZAR, 1996).

\section{RESULTADOS}

A única diferença entre os grupos foi o maior valor de $\mathrm{PaO}_{2}$ aos 40 minutos de anestesia no GPC e menor valor de glicose nesse mesmo grupo aos 10 minutos após a MPA. Quando comparada à evolução dos parâmetros ao longo do tempo dentro de cada grupo em relação ao valor basal, nos animais do GP, a FC reduziu apenas aos 10 minutos após a MPA, e a PAM de 20 a 40 minutos durante a anestesia (Tabela 1).

A ETCO ${ }_{2}$ e o bicarbonato aumentaram após 10 minutos durante a anestesia com propofol/cetamina e a $\mathrm{PaCO}_{2}$ aumentou em ambos os grupos entre 20 e 30 minutos de anestesia. $\mathrm{O} \mathrm{pH}$ reduziu em ambos os grupos a partir de 10 minutos da indução até o final da anestesia. $\mathrm{A} \mathrm{PaO}_{2}$ aumentou no GPC apenas aos 30 minutos de anestesia e a glicose entre 20 e 40 minutos de anestesia (Tabela 1). Três cadelas do GP e duas do GPC apresentaram apnéia durante a anestesia. Esta foi prontamente revertida pelo estímulo doloroso cirúrgico.

A temperatura reduziu após 10 minutos da anestesia em ambos os grupos. Entretanto os valores sempre foram superiores a $37^{\circ} \mathrm{C}$ (Tabela 1 ).

A escala analógica visual avaliada pelo cirurgião foi similar entre os grupos, porém houve necessidade de incrementar em $50 \%$ e $20 \%$ a taxa de infusão do propofol nos grupos GP e GPC, respectivamente, para promover anestesia satisfatória para a realização do procedimento cirúrgico. Dessa forma as taxas de manutenção de propofol foram 0,6 e 0,34mg $\mathrm{kg}^{-1} \cdot \mathrm{min}^{-1}$ nos grupos GP e GPC, respectivamente.

Os animais assumiram a posição quadrupedal em $37 \pm 2$ e $28 \pm 3$ minutos após o término da infusão de propofol ou cetamina/propofol, respectivamente (Tabela 2 ).

\section{DISCUSSÃO}

O propofol exerce efeitos discretos sobre o ritmo cardíaco (LÓPEZ et al., 1994). Porém, pode ocorrer 
Tabela 1 - Valores médios e desvio padrão da frequiência cardíaca (FC), pressão arterial média (PAM), saturação de oxigênio na hemoglobina $\left(\mathrm{SatO}_{2}\right)$, concentração final expirada de $\mathrm{CO}_{2}\left(\mathrm{ETCO}_{2}\right)$, volume minuto $(\mathrm{VM})$, freqüência respiratória $(f), \mathrm{pH}$, bicarbonato $(\mathrm{mEq} / \mathrm{l})$, pressão parcial de gás carbônico $\left(\mathrm{PaCO}_{2}\right)$, pressão parcial de oxigênio $\left(\mathrm{PaO}_{2}\right)$, glicose $(\mathrm{g} / \mathrm{dl})$ e temperatura retal (T) em cadelas pré-tratadas com atropina e xilazina e anestesiadas com propofol (GP, n=6) ou propofol/cetamina (GPC, $\mathrm{n}=6)$.

\begin{tabular}{|c|c|c|c|c|c|c|c|}
\hline momentos & Basal & 10min MPA & Pós indução & $\begin{array}{l}\text { 10min } \\
\text { indução }\end{array}$ & $\begin{array}{l}\text { 20min } \\
\text { indução }\end{array}$ & $\begin{array}{c}\text { 30min } \\
\text { indução }\end{array}$ & $\begin{array}{l}\text { 40min } \\
\text { indução }\end{array}$ \\
\hline \multicolumn{8}{|c|}{ FC (bat $\left.\min ^{-1}\right)$} \\
\hline GP & $143 \pm 12^{\mathrm{A}}$ & $101 \pm 6^{\mathrm{B}}$ & $133 \pm 9^{\mathrm{AB}}$ & $126 \pm 8^{\mathrm{AB}}$ & $124 \pm 7^{\mathrm{AB}}$ & $115 \pm 7^{\mathrm{AB}}$ & $121 \pm 7^{\mathrm{AB}}$ \\
\hline GPC & $131 \pm 13$ & $111 \pm 19$ & $115 \pm 11$ & $108 \pm 13$ & $108 \pm 13$ & $117 \pm 19$ & $125 \pm 13$ \\
\hline \multicolumn{8}{|c|}{ PAM (mmHg) } \\
\hline GP & $133 \pm 10^{\mathrm{A}}$ & $124 \pm 10^{\mathrm{A}}$ & $105 \pm 10^{\mathrm{AB}}$ & $96 \pm 4^{\mathrm{AB}}$ & $89 \pm 6^{\text {B }}$ & $86 \pm 8^{\text {B }}$ & $81 \pm 7^{\text {В }}$ \\
\hline GPC & $110 \pm 12$ & $111 \pm 7$ & $102 \pm 10$ & $94 \pm 9$ & $83 \pm 10$ & $92 \pm 7$ & $103 \pm 10$ \\
\hline \multicolumn{8}{|l|}{$\mathrm{SatO}_{2}(\%)$} \\
\hline GP & $97 \pm 0,5$ & $96 \pm 1$ & $96 \pm 1$ & $97 \pm 1$ & $96 \pm 1,5$ & $96 \pm 1$ & $96 \pm 1$ \\
\hline GPC & $96 \pm 1$ & $95 \pm 3$ & $97 \pm 1$ & $94 \pm 4$ & $95 \pm 4$ & $96 \pm 2$ & $98 \pm 1$ \\
\hline \multicolumn{8}{|c|}{$\mathrm{ETCO}_{2}(\mathrm{mmHg})$} \\
\hline GP & $39 \pm 5$ & $41 \pm 2$ & $44 \pm 2$ & $48 \pm 3$ & $49 \pm 5$ & $48 \pm 5$ & $46 \pm 3$ \\
\hline GPC & $36 \pm 3^{A}$ & $37 \pm 2^{\mathrm{A}}$ & $45 \pm 4^{\mathrm{AB}}$ & $51 \pm 2^{\text {B }}$ & $50 \pm 3^{\text {B }}$ & $49 \pm 3^{\text {B }}$ & $43 \pm 3^{\mathrm{AB}}$ \\
\hline \multicolumn{8}{|c|}{ VM $\left(1 . \min ^{-1}\right)$} \\
\hline GP & $7,2 \pm 1,5$ & $4 \pm 1$ & $2,5 \pm 0,5$ & $2,7 \pm 1$ & $3 \pm 1$ & $3,1 \pm 1$ & $4 \pm 1$ \\
\hline GPC & $4,5 \pm 7$ & $2,8 \pm 7$ & $2,8 \pm 7$ & $4,3 \pm 1,5$ & $4,3 \pm 1$ & $5,5 \pm 1$ & $8,3 \pm 2$ \\
\hline \multicolumn{8}{|c|}{$f\left(\right.$ mov.min $\left.^{-1}\right)$} \\
\hline GP & $29 \pm 5$ & $27 \pm 8$ & $16 \pm 6$ & $16 \pm 3$ & $36 \pm 24$ & $19 \pm 6$ & $26 \pm 7$ \\
\hline GPC & $20 \pm 3$ & $14 \pm 1$ & $14 \pm 2$ & $27 \pm 7$ & $30 \pm 9$ & $32 \pm 8$ & $38 \pm 7$ \\
\hline \multicolumn{8}{|l|}{$\mathrm{pH}$} \\
\hline GP & $7,38 \pm 0,2^{\mathrm{A}}$ & $7,39 \pm 0,1^{\mathrm{A}}$ & ---- & $7,28 \pm 0,1^{\text {B }}$ & $7,25 \pm 0,1^{\text {B }}$ & $7,25 \pm 0,1^{\text {B }}$ & $7,27 \pm 0,1^{\text {B }}$ \\
\hline GPC & $7,41 \pm 0,1^{\mathrm{A}}$ & $7,42 \pm 0,1^{\mathrm{A}}$ & ----- & $7,28 \pm 0,1^{\mathrm{B}}$ & $7,24 \pm 0,1^{\mathrm{B}}$ & $7,26 \pm 0,1^{\mathrm{B}}$ & $7,27 \pm 0,1^{\text {B }}$ \\
\hline \multicolumn{8}{|c|}{ Bicarbonato (mEq/l) } \\
\hline GP & $17 \pm 1$ & $19 \pm 1$ & ----- & $19 \pm 1$ & $20 \pm 1$ & $20 \pm 1$ & $20 \pm 1$ \\
\hline GPC & $17 \pm 1^{\mathrm{A}}$ & $19 \pm 1^{\mathrm{AB}}$ & ---- & $20 \pm 1^{\mathrm{B}}$ & $22 \pm 1^{\mathrm{B}}$ & $22 \pm 1^{\mathrm{B}}$ & $22 \pm 1^{\mathrm{B}}$ \\
\hline \multicolumn{8}{|c|}{$\mathrm{PaCO}_{2}(\mathrm{mmHg})$} \\
\hline GP & $28 \pm 3^{A}$ & $31 \pm 2^{\mathrm{AB}}$ & ---- & $44 \pm 4^{\mathrm{AB}}$ & $49 \pm 6^{\mathrm{B}}$ & $47 \pm 5^{\mathrm{B}}$ & $45 \pm 4^{\mathrm{AB}}$ \\
\hline GPC & $27 \pm 2^{\mathrm{A}}$ & $29 \pm 2^{\mathrm{AB}}$ & ---- & $44 \pm 6^{\mathrm{AB}}$ & $50 \pm 6^{\text {B }}$ & $48 \pm 4^{\text {B }}$ & $47 \pm 4^{\mathrm{B}}$ \\
\hline \multicolumn{8}{|c|}{$\mathrm{PaO}_{2}(\mathrm{mmHg})$} \\
\hline GP & $92 \pm 4$ & $81 \pm 8$ & ----- & $146 \pm 37$ & $171 \pm 48$ & $146 \pm 34$ & $125125 \pm 31^{\mathrm{a}}$ \\
\hline GPC & $86 \pm 6^{\mathrm{A}}$ & $91 \pm 11^{\mathrm{A}}$ & ----- & $235 \pm 54^{\mathrm{AB}}$ & $255 \pm 64^{\text {B }}$ & $256 \pm 48^{\mathrm{B}}$ & $260 \pm 46^{\mathrm{Bb}}$ \\
\hline \multicolumn{8}{|c|}{ Glicose (g/dl) } \\
\hline GP & $108 \pm 9$ & $114 \pm 6^{\mathrm{a}}$ & ----- & $170 \pm 34$ & $192 \pm 41$ & $198 \pm 43$ & $205 \pm 41$ \\
\hline GPC & $89 \pm 5^{\mathrm{A}}$ & $93 \pm 5^{\mathrm{Ab}}$ & ----- & $139 \pm 16^{\mathrm{AB}}$ & $162 \pm 21^{\mathrm{B}}$ & $169 \pm 23^{\mathrm{B}}$ & $172 \pm 24^{\mathrm{B}}$ \\
\hline \multicolumn{8}{|l|}{$\mathrm{T}\left({ }^{\circ} \mathrm{C}\right)$} \\
\hline GP & $39,3 \pm 0,2^{\mathrm{A}}$ & $39,1 \pm 0,2^{\mathrm{A}}$ & $38,5 \pm 0,2^{\mathrm{AB}}$ & $38,1 \pm 0,1^{\text {B }}$ & $37,8 \pm 0,1^{\mathrm{B}}$ & $37,6 \pm 0,1^{\text {B }}$ & $37,5 \pm 0,1^{\text {B }}$ \\
\hline GPC & $39,5 \pm 0,2^{\mathrm{A}}$ & $39,3 \pm 0,2^{\mathrm{A}}$ & $38,7 \pm 0,3^{\mathrm{AB}}$ & $38,2 \pm 0,2^{\mathrm{B}}$ & $37,7 \pm 0,2^{\mathrm{B}}$ & $37,2 \pm 0,3^{\mathrm{B}}$ & $37,1 \pm 0,3^{\mathrm{B}}$ \\
\hline
\end{tabular}

Dentro de cada grupo, momentos seguidos de letras maiúsculas diferentes na mesma linha diferem estatisticamente. Diferenças entre os grupos em cada momento estão expressas em letras minúsculas diferentes, na mesma coluna. $\mathrm{P}<0,05$.

bradiarritmia secundária à menor sensibilidade barorreflexa, induzida pela inibição da atividade simpática (BRANSON, 2007). Neste estudo, não foram detectadas alterações de ritmo e freqüência cardíaca em nenhum dos grupos avaliados durante o procedimento anestésico. Ocorreu discreta redução da freqüência cardíaca em ambos os grupos durante o procedimento anestésico, dentro dos limites fisiológicos para a espécie, o que corrobora os resultados prévios relatados em cães submetidos à infusão contínua de propofol ou propofol/cetamina, durante 60 minutos (MANNARINO, 2002; MANNARINO, 2005). A medicação pré-anestésica com xilazina pode ter contribuído para a redução da 
Tabela 2 - Valores médios e desvio padrão do tempo decorrido entre o término da anestesia até o animal conseguir levantar a cabeça, manter-se em decúbito esternal e em posição quadrupedal e escala analógica visual (EAV) quanto à qualidade de anestesia cirúrgica e relaxamento muscular em cadelas pré-tratadas com atropina e xilazina e anestesiadas com propofol (GP, $\mathrm{n}=6$ ) ou propofol/cetamina (GPC, n=6).

\begin{tabular}{lllll}
\hline Grupos & cabeça & esternal & estação & EAV \\
\hline GP & $13 \pm 2$ & $17 \pm 2$ & $37 \pm 2$ & $8,5 \pm 0,4$ \\
GPC & $9 \pm 2$ & $20 \pm 3$ & $28 \pm 3$ & $8,6 \pm 0,5$ \\
\hline
\end{tabular}

freqüência cardíaca, pois esse fármaco induz depressão cardiovascular (WRIGHT, 1982). Entretanto o tratamento com atropina possivelmente evitou a bradicardia. Embora tenha sido observada redução significativa na FC após a MPA nos animais do GP, esse achado não apresenta relevância clínica, uma vez que essa variável manteve-se dentro dos limites fisiológicos para a espécie canina (HASKINS, 2007).

Redução significativa da pressão arterial média (PAM) foi identificada nos animais tratados apenas com propofol (GP). No entanto, deve-se ressaltar que nesse grupo, embora sem diferença significativa, os valores basais foram superiores em relação ao grupo tratado com propofol/cetamina (GPC). Dessa forma, aos 20 minutos após a indução anestésica, apesar de ter sido observada redução significativa da PAM no grupo GP, deve-se salientar que o valor registrado foi superior ao do GPC, de modo que tal achado não apresentou relevância clínica, visto que os grupos não eram homogêneos em relação aos valores basais. Portanto, embora discreta redução da PAM tenha sido detectada em ambos os grupos, os valores ficaram dentro dos limites fisiológicos, compreendidos entre $80-120 \mathrm{mmHg}$ (HASKINS, 2007). Resultados divergentes foram relatados por SELISKAR et al. (2007), os quais observaram valores superiores de PAM em cães anestesiados com propofol/cetamina em relação ao propofol isolado. No entanto, no presente estudo, além de anestesiados os animais foram submetidos à ovariossalpingohisterectomia, sugerindo que o estímulo nociceptivo cirúrgico tenha promovido ativação simpática, traduzida pela liberação de catecolaminas, atenuando o efeito depressor simpático induzido pelo propofol.

A depressão respiratória foi observada em ambos os grupos, achado que corrobora os resultados previamente relatados na espécie canina (SELISKAR et al., 2007; INTELIZANO et al., 2008). O propofol induz depressão respiratória, com redução do volume minuto, aumento da $\mathrm{PaCO}_{2}$ eETCO 2 e redução do $\mathrm{pH}$ sanguíneo
(INTELIZANO et al., 2008). A cetamina pode favorecer a depressão respiratória por desencadear a respiração apnêustica e aumentar a $\mathrm{PaCO}_{2}$ (MUIR \& GADAWSKI, 1998). Desse modo, suplementação com oxigênio a $100 \%$ é fundamental, bem como o suporte de ventilação artificial, necessário em ambos os grupos (VIEIRA, 1999; MANARINO, 2002; INTELIZANO et al., 2008).

A redução progressiva da temperatura retal em ambos os grupos ocorreu em função de diversos fatores, como vasodilatação periférica, redução do metabolismo basal, aumento da perda de calor e depressão do centro termorregulador, classicamente observados durante a anestesia (KISSIN, 1993). Entretanto os valores nunca foram inferiores a $37^{\circ} \mathrm{C}$, mostrando a importância e efetividade do aquecimento artificial por meio de colchão térmico.

A hiperglicemia observada em ambos os grupos foi provavelmente induzida pela xilazina, em função de hipoinsulinemia, decorrente da estimulação $\alpha$-adrenérgica sobre as células $\beta$ do pâncreas (PADDLEFORD \& HARVEY, 1999). Além disso, durante procedimentos cirúrgicos e anestésicos, a resposta de estresse aumenta a concentração plasmática de glicose, em função do incremento da liberação de catecolaminas e cortisol (MARANA et al., 2000). Embora sem diferença significativa, a glicemia apresentou-se mais elevada nos animais anestesiados com propofol, quando comparados aos anestesiados com propofol e cetamina, sugerindo que a resposta de estresse foi menos evidente no grupo GPC, em função das propriedades analgésicas da cetamina, que atua antagonizando os receptores NMDA (ANGEL \& GRATTON, 1982), de modo a promover efeitos antinociceptivos capazes de reduzir o estímulo induzido pelo ato cirúrgico sobre o eixo hipófise-pituitária-adrenal (MARANA et al., 2000).

Embora a escala analógica visual avaliada pelo cirurgião tenha sido similar entre os grupos, devese ressaltar que essa observação é conseqüência do incremento de propofol realizado no período transoperatório, visando à obtenção de plano anestésico adequado para o procedimento cirúrgico. Dessa forma, a qualidade da anestesia foi superior nos animais anestesiados com propofol/cetamina, visto que a necessidade de incremento na infusão do propofol foi de $20 \%$, enquanto nos animais anestesiados apenas com propofol foi de $50 \%$, corroborando os resultados relatados por INTELIZANO et al. (2008).

A recuperação anestésica foi tranqüila, sem sinais de excitação e relativamente rápida quando comparada a estudos similares. VIEIRA (1999) e MANNARINO (2002) observaram recuperação prolongada em cadelas submetidas à OSH, porém esses pesquisadores utilizaram taxas de infusão de propofol 
bem superiores $\left(1,5\right.$ e $1,25 \mathrm{mg} \mathrm{kg}^{-1} \cdot \mathrm{min}^{-1}$, respectivamente) às taxas utilizadas neste estudo, o que demonstra o efeito dose-dependente do propofol, em função do acúmulo desse fármaco em tecidos periféricos (ESTIVALET et al., 2002). Além disso, o tempo de recuperação não variou entre os grupos, corroborando os resultados relatados por VIEIRA (1999), com o uso de propofol isolado ou associado à cetamina.

Para viabilizar um plano anestésico adequado, houve necessidade de aumentar a taxa de infusão de propofol em ambos os grupos. Assim, salienta-se que há necessidade de adequar a taxa de infusão de acordo com a necessidade de cada indivíduo e o plano cirúrgico requerido. Paralelamente, ressaltase a necessidade de intervir com ventilação artificial a fim de manterem-se estáveis as variáveis respiratórias, visto que ambos os protocolos determinam depressão respiratória pronunciada.

\section{CONCLUSÕES}

Perante as condições experimentais desse estudo, conclui-se que, tanto a infusão de propofol, quanto a infusão de cetamina/propofol promoveram estabilidade cardiovascular e anestesia cirúrgica satisfatória para realização de OSH em cadelas, desde que a taxa de infusão seja ajustada em função do estímulo nociceptivo cirúrgico, de modo a determinar plano anestésico adequado para a realização do procedimento.

\section{FONTES DE AQUISIÇÃO}

a-Sulfato de atropina 0,25mg, Ariston Ind. Quim e Farms. Ltda, São Paulo, Brasil.

b-Coopazine 2\%, Coopers, São Paulo Brasil.

c-Diprivan 1\%, Zeneca Farmacêutica do Brasil Ltda, São Paulo, Brasil.

d-Ketalar 5\%, Parke Davis Warner Lambert, São Paulo, Brasil e-ANNE Anesthesia Infuser, Abbott Brasil, São Paulo, Brasil. f-Monitor 4-1CN e ECG-5, Ecafix Funbec, São Paulo, Brasil. g-Becton Dickinson Indústrias Farmacêuticas Ltda, São Paulo, Brasil.

h-DX7100, Dixtal Biomédica Indústria e Comércio Ltda, Manaus, AM, Brasil.

i-Ventilômetro, Oxygel, São Paulo, Brasil.

j-DX2710, Dixtal Biomédica Indústria e Comércio Ltda, Manaus, AM, Brasil.

k-NT500-1, Microtherm do Brasil, São Paulo, Brasil.

l-pH/Blood Gas Analyzer, model 248, Ciba Corning Diagnostics, Medfield, MA, USA.

m-Insyte, Becton Dickinson, Juiz de Fora, MG, Brasil.

\section{COMITÊ DE ÉTICA}

Este estudo foi aprovado pela Câmara de Ética em Experimentação Animal (CEEA) da Faculdade de Medicina
Veterinária e Zootecnia, Universidade Estadual Paulista (UNESP), Botucatu, sob o protocolo n.27/2008, realizado de acordo com os princípios éticos na experimentação animal.

\section{REFERÊNCIAS}

ANGEL, A.; GRATTON, D.A. The effects of anaesthetic agents on cerebral cortical response in the rat. British Journal of Pharmacology, v.76, 541-549, 1982.

BRANSON, K.R. Injectable and alternative anesthetics techniques. In: Tranquilli, W.J.; Thurmon, J.C.; Grimm, K.A. Lumb \& Jones' Veterinary Anesthesia and Analgesia.4. ed. Oxford: Blackwell Publishing, 2007, Cap. 11, p.273-300.

BULAFARI, A. et al. A comparative study of neurologicallyequivalent propofol anaesthetic combinations in the dog. Journal of Veterinary Anaesthesia, v.22, p.19-24, 1995.

COURT, M.H. et al. Cytocrome P-450 2B6 is responsible for interindividual variability of propofol hydroxylation by human liver microssomes. Anesthesiology, v.94, p.110-119, 2001.

CULLEN, L.K.; REYNOLDSON, J.A. Xylazine or medetomidine premedication before propofol anaesthesia. Veterinary Record, v.132, p. 378-383, 1993.

ENGLAND, G.C. et al. Romifidine as a premedicant to propofol induction and infusion anaesthesia in the dog. Journal of Small Animal Practice, v.37, p.79-83, 1996.

ESTIVALET, F.F. et al. Remifentanil associado ao propofol ou sevoflurano para a colecistectomia videolaparoscópica. Estudo comparativo. Revista Brasileira de Anestesiologia, v.52, p.385-393, 2002.

GANEM, E.M. et al. Eficácia do propofol e da associação de propofol e dexametasona no controle da náusea e vômito no pós-operatório de laparoscopia ginecológica. Revista Brasileira de Anestesiologia, v.52, p.394-401, 2002.

HALL, L.W.; CHAMBERS, J.P. A clinical trial of propofol infusion anesthesia in dogs. Journal of Small Animal Practice, v.28, p.623-37, 1987.

HASKINS, S.C. Monitoring anesthetized patients. In: Tranquilli, W.J.; Thurmon, J.C.; Grimm, K.A. Lumb \& Jones' Veterinary Anesthesia and Analgesia.4. ed. Oxford: Blackwell Publishing, 2007, Cap. 19, p.533-560.

HATSCHBACH, E. et al. Comparative study between targetcontrolled-infusion and continuous-infusion anesthesia in dogs treated with methotrimeprazine and treated with propofol and remifentanil. Acta Cirúrgica Brasileira, v.23, p. 65-72, 2008.

HEMERIJAK, J.V.; KISSIN, I. História da Anestesia Venosa. In: WHITE, P.F. Tratado de Anestesia Venosa. Porto Alegre: Artmed Editora, 2001. Cap.1, p.19-26

ILKIW, J.E. Balanced anesthetic techniques in dogs and cats. Clinical Techniques in Small Animal Practice, v.14, p. 27-37, 1999.

INTELIZANO, T.R. et al. Total intravenous anaesthesia with propofol-racemic ketamine and propofol-S-ketamine: A 
comparative study and haemodynamic evaluation in dogs undergoing ovariohysterectomy. Pesquisa Veterinária Brasileira, v. 28, p.216-222, 2008.

KISSIN, I. General anaesthetic action: obsolete notion? Anesthesia and Analgesia, v.76, p.215-218, 1993.

LÓPEZ, H.S. et al. Anestesia general con propofol en perros mediante infusión continua. Experiencias clínicas. Veterinaria Mexico, v.25, p.199-205, 1994.

MANNARINO, R. Determinação da taxa de infusão mínima de propofol e propofol associado à lidocaína em cães (Cannis familiaris). Botucatu, 2002. 128p. Dissertação (Mestrado em Anestesiologia) - Faculdade de Medicina de Botucatu, Universidade Estadual Paulista.

MANNARINO, R. Determinação das taxas de infusões mínimas e estudo hemodinâmico, respiratório, metabólico, e farmacocinético do propofol e de sua associação com lidocaína e cetamina em cães. Botucatu, 2005. 128p. Tese (Doutorado em Anestesiologia) - Faculdade de Medicina de Botucatu, Universidade Estadual Paulista.

MARANA, R. et al. Stress responses to endoscopic surgery. Current Opinion in Obstetrics \& Gynecology, v.12, p.303-307, 2000.

MASSONE, F. Anestesiologia veterinária: farmacologia e técnicas. 5 ed. Rio de Janeiro, Guanabara Koogan, 2008. 571p.

MUIR, W.W. III. Balanced anaesthesia: new emphasis on an old idea. Journal of the American Veterinary Medical Association, v.21, p.9-11, 1994.

MUIR, W.W. III; GADAWSKI, J.E. Respiratory depression and apnea induced by propofol in dogs. American Journal Veterinary Research, v.59, p.157-161, 1998.
O’CONNOR, M.; SEAR, J.W. Sufentanil to supplement nitrous oxide in oxygen during balanced anaesthesia. Anaesthesia, v.43, p.749-752, 1988.

PADDLEFORD, R.R.; HARVEY, R.C. Alpha agonists and antagonists. Veterinary Clinics of North America: Small Animal Practice, v.29, p.737-745, 1999.

REDONDO, J.I. et al. Romifidine, medetomidine or xylazine before propofol-halothane- $\mathrm{N}_{2} \mathrm{O}$ anesthesia in dogs. Canadian Journal of Veterinary Research, v.63, p.31-36, 1999.

SELISKAR, A. et al. Total intravenous anaesthesia with propofol or propofol/ketamine in spontaneously breathing dogs premedicated with medetomidine. Veterinary Record, v.160, p.85-91, 2007.

SMITH, I. et al. Propofol: an update on its clinical use. Anesthesiology, v.81, p.1005-1043, 1994.

VIEIRA, F.A.F. Comparação entre propofol e propofol/ cetamina na indução e manutenção anestésica por infusão contínua intravenosa após medicação préanestésica com levomepromazina em cães. Botucatu, 1999. 123p. Dissertação (Mestrado em Cirurgia) - Faculdade de Medicina Veterinária e Zootecnia, Universidade Estadual Paulista.

ZAR, J. H. In: Biostatistical analysis. New Jersey: PrenticeHall Upper Saddle River, 1996. p.718.

WATKINS, S.B. et al. Propofol as an intravenous anesthetic agent in dogs. Veterinary Record, v.120, p.326-329, 1987.

WRIGHT, M. Pharmacologic effects of ketamine and its use in veterinary medicine. Journal of the American Veterinary Medical Association, v.180, p.1462-1470, 1982. 